\title{
Lymphangiogenesis and biological behavior in pancreatic carcinoma and other pancreatic tumors
}

\author{
ZHONGQIU WANG $^{1 *}$, JIANG WU ${ }^{1 *}$, GUOJUN LI $^{3}$, XINHUA ZHANG ${ }^{2}$, \\ MINGMIN TONG ${ }^{1}$, ZHENGCAN WU ${ }^{1}$ and ZHENJUAN LIU ${ }^{1}$
}

\author{
Departments of ${ }^{1}$ Medical Imaging, and ${ }^{2}$ Pathology, Jinling Hospital, Medical School of \\ Nanjing University, Nanjing 210002, P.R. China; ${ }^{3}$ Department of Head and Neck Surgery \\ and Epidemiology, MD Anderson Cancer Center, Houston, TX 77030, USA
}

Received September 5, 2011; Accepted December 30, 2011

DOI: $10.3892 / \mathrm{mmr} .2012 .745$

\begin{abstract}
Lymphatic vessels in primary tumor tissue play an important role in lymphatic metastasis. Lymphatic metastasis of malignant neoplasms is significantly related to prognosis, influencing both recurrence and survival. The aim of this study was to investigate the correlation of intra-tumoral lymphatic vessel density (iLVD) and peri-tumoral lymphatic vessel density (pLVD) with biological behavior and prognostic parameters in pancreatic carcinoma (PC) and other pancreatic tumors. Lymphangiogenesis was examined using the D2-40 monoclonal antibody in 33 cases of PC, 7 neuroendocrine tumors of the pancreas (NETP), 7 solid pseudopapillary tumors of the pancreas (SPTP) and 3 cystadenomas of the pancreas (CP). Positively-stained microvessels were counted at magnification $x 400$ in dense lymphatic vascular foci (hotspots). The LVD of PC was compared to 3 other pancreatic tumors. The relationships among the LVD, the extent of differentiation, lymphatic invasion, lymph node metastasis and other clinicopathological parameters of $\mathrm{PC}$ were analyzed. There was no difference in the iLVD among PC, NETP, SPTP and CP. The pLVD of NETP was markedly higher than that of PC, SPTP and CP. The pLVD of PC was significantly higher than that of SPTP and CP, but there was no difference between SPTP and CP. The PLVD of PC was significantly associated with the extent of differentiation, lymphatic invasion and lymph node metastasis, whereas it was not associated with age, gender, tumor size, tumor location and peri-pancreatic invasion. The
\end{abstract}

Correspondence to: Dr Zhongqiu Wang, Department of Medical Imaging, Jinling Hospital, Medical School of Nanjing University, Nanjing 210002, P.R. China

E-mail: zhq2001us@yahoo.com

*Contributed equally

Abbreviations: iLVD, intra-tumoral lymphatic vessel density; pLVD, peri-tumoral lymphatic vessel density; PC, pancreatic carcinoma; NETP, neuroendocrine tumor of the pancreas; SPTP, solid pseudopapillary tumor of the pancreas; $\mathrm{CP}$, cystadenoma of the pancreas

Key words: pancreatic tumor, D2-40, lymhangiogenesis, metastasis
iLVD of PC was not correlated with these clinicopathological parameters. There was no difference in iLVD and no marked difference in pLVD among the pancreatic tumors. Detection of pLVD is of greater importance than detecting iLVD in these pancreatic tumors, as pLVD can be utilized for the prediction of lymph node metastasis, thus aiding in the evaluation of patient prognosis.

\section{Introduction}

Invasion and metastasis are the cause of mortality in approximately $80 \%$ of tumor patients. Metastasis occurs mainly through vascular and lymphatic vessels (1-7). Research into the mechanism of tumor metastasis has primarily focused on vascular vessels, with little focus on lymphatic vessels. Lymphatic vessels are important pathways for metastasis and are a primary cause of post-operative tumor recurrence $(1,3,6,8)$. Therefore, it is of great clinical and physiological significance to discover the molecular mechanisms underlying tumor development and metastasis through lymphatic vessels and to explore ways of preventing tumor lymphatic metastasis. The purpose of this study was to examine the relationship between tumor lymphatic vessel development, tumor metastasis and patient prognosis. The monoclonal antibody D2-40 was used immunohistochemically to detect the lymphatic vessel density (LVD) in the pancreatic tumors - pancreatic carcinoma (PC), neuroendocrine tumors of the pancreas (NETP), solid pseudopapillary tumors of the pancreas (SPTP) and cystadenomas of the pancreas (CP). The results of LVD and the clinical and pathological parameters of the tumors were compared and analyzed.

\section{Materials and methods}

Subjects. Fifty specimens of pancreatic tumor tissues were obtained from the Department of Pathology, Jinling Hospital, Medical School of Nanjing University. The tumor samples were obtained from surgical resections. Of the 50 cases, 33 were of PC (male 24; female 9; age range 38-79 years; average 64.6), 7 of NETP (male 3; female 4; age range 39-61 years; average 52; pathological division as follows: 3 cases of carcinoid, 2 cases of insulinoma, 2 cases of NETP without being further 

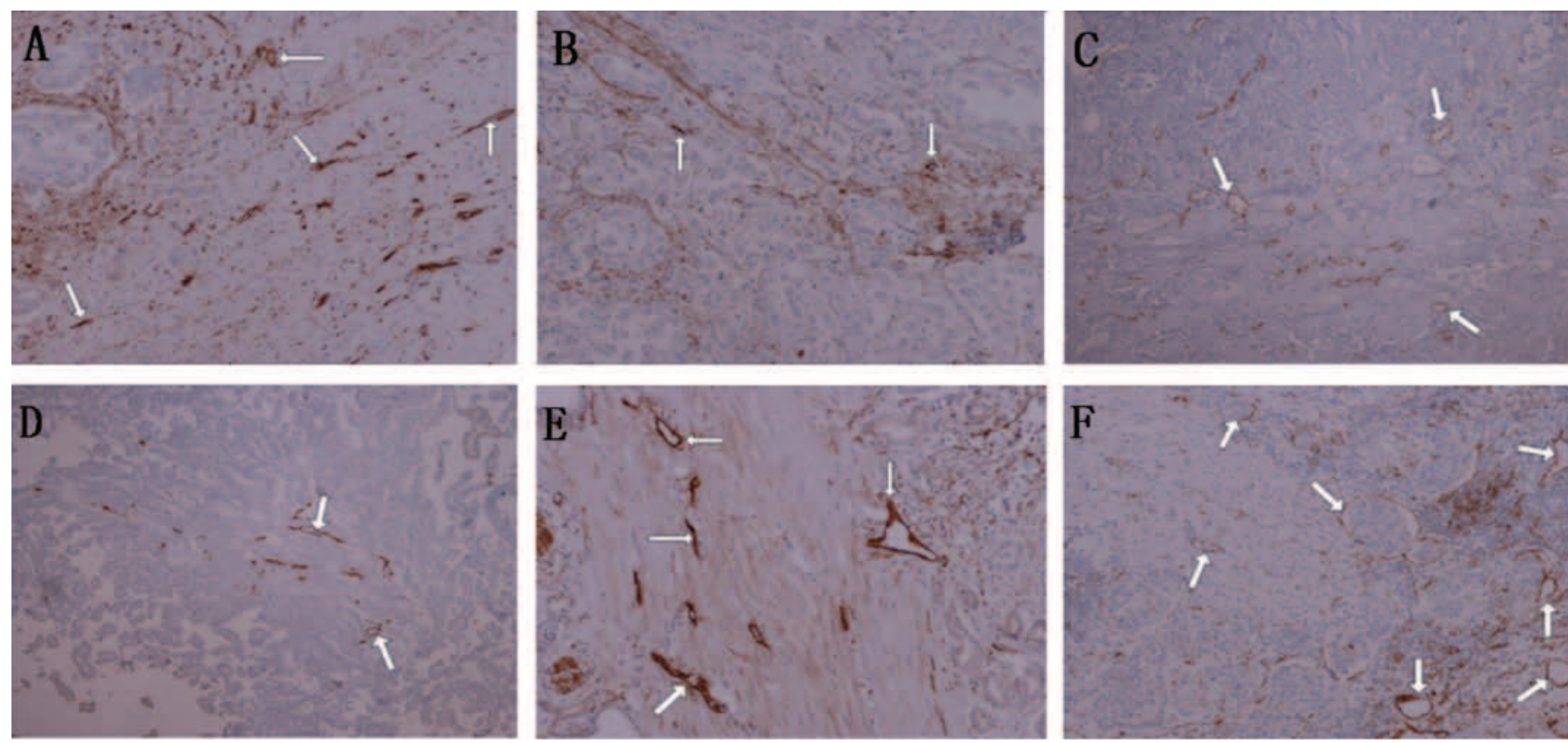

Figure 1. Histological and immunohistochemical characteristics of the pancreatic tumor tissues. The brown lymph vessels (white arrows) in the peri-tumor tissue of pancreatic cancer (PC). (B) The same patient as in (A). The intra-tumoral lymphatic vessel density (iLVD) (white arrows) of PC is significantly lower than the peri-tumoral lymphatic vessel density (pLVD) in (A). (C) The pLVD of PC. (D) The pLVD of SPTP is significantly lower than that in (C). (E) The pLVD in high and moderate PC. (F) The pLVD in poor PC is significantly higher than that in (E). The cancer embolus is observed in lymphatic vessel (white arrow). All of the above were carried out by D2-40 immunohistochemistry with low magnification microscopy; $x 100$.

classified), 7 of SPTP (male 1; female 6; age range 23-69 years; average 32.1), and 3 were of CP (all female; age 38, 39 and 38 years; pathological division as follows: 2 cases of serous cystadenoma and 1 case of mucinous cystadenoma).

Detection technique. Immunohistochemistry was carried out with EnVision, using the monoclonal antibody D2-40 (American Dako Co.) and the EnVision reagent kit (Fuzhou Maixing Biology Technique Developmental Ltd. Co.). The operate process was performed by illuminate book. Repairing antigen with autoclave, DAB coloration, neutrality gum envelop slice and tissue slice were observed under microscope.

Analysis of the results. Firstly, peri-tumoral and intra-tumoral areas of the pancreatic tumors were observed by low magnification microscopy ( $x 40$ or $x 100)$, choosing the area with the highest LVD. The lymphatic vessels were dyed a brown color. Secondly, the number of brown lymphatic vessels in the area was observed by high magnification microscopy (x400). The average number of lymphatic vessels in five areas (hotspots) was then calculated $(2,9)$. Therefore, the peri-tumoral lymphatic vessel density (pLVD) and intra-tumoral lymphatic vessel density (iLVD) in the pancreatic tumors were calculated according to this method.

Statistical analysis. The data are expressed as the mean \pm standard deviation (SD). The mean values of the two samples were compared with the Student's t-test. The mean values among multi-samples were compared using analysis of variance. SPSS 10.0 software was used for data analysis and $\mathrm{P}<0.05$ was considered to denote statistical significance.

\section{Results}

Characteristics of lymphatic vessels. As shown in Fig. 1, the brown lymphatic vessels were noted in dyed position D2-40 areas. The brown regions were found to be located in the cell membrane and cytoplasm in the inner cell of the lymphatic vessel. The wall of the lymphatic vessels was relatively thin, composed of multi single-slice inner cells. The form of these lymphatic vessels was irregular, dilative, circular, polygonal or occlusive strip. The negative dyed vessels were vascular. Some red cells were observed in the lumen of the vascular vessels.

Comparison of LVD among PC, NETP, SPTP and CP. Comparisons of pLVD and iLVD in PC, NETP, SPTP and CP are shown in Table I. Of the four groups of pancreatic tumors, the pLVD was highest in NETP. The statistical difference was apparent $(\mathrm{P}=0.000)$. The pLVD of $\mathrm{PC}$ was higher than that of SPTP and CP. The statistical difference was also evident $(\mathrm{P}<0.05)$. No statistical difference was identified in pLVD between SPTP and CP (Table II). Likewise, there was no statistical difference in iLVD between any two groups among the four (Table III).

Relationship between the PLVD and iLVD of PC and clinicopathological parameters. The pLVD of PC was found to be related to degree of differentiation, lymphatic node metastasis and lymphatic vessel invasion $(\mathrm{P}<0.05)$, but not to age or the gender of the patients, nor the tumor size, tumor location or invasiveness of the tumor. The iLVD of PC was not related to any clinicopathological parameters (Fig. 2). 
Table I. Comparison of pLVD and iLVD in PC, NETP, SPTP and CP.

\begin{tabular}{|c|c|c|c|c|c|c|c|}
\hline Pancreatic tumor & $\mathrm{n}$ & pLVD $($ mean \pm SD $)$ & $\mathrm{F}$ & P-value & iLVD $($ mean $\pm S D)$ & $\mathrm{F}$ & P-value \\
\hline $\mathrm{PC}$ & 33 & $6.661 \pm 1.193$ & & & $1.552 \pm 1.207$ & & \\
\hline \multirow[t]{2}{*}{ NETP } & 7 & $12.343 \pm 3.442$ & & & $3.714 \pm 2.360$ & & \\
\hline & & & 23.419 & 0.000 & & 5.854 & 0.002 \\
\hline SPTP & 7 & $3.657 \pm 1.735$ & & & $0.971 \pm 0.927$ & & \\
\hline $\mathrm{CP}$ & 3 & $2.733 \pm 1.555$ & & & $1.333 \pm 0.833$ & & \\
\hline
\end{tabular}

PC, pancreatic carcinoma; NETP, neuroendocrine tumor of the pancreas; SPTP, solid pseudopapillary tumor of the pancreas; CP, cystadenoma of the pancreas; pLVD, peri-tumoral lymphatic vessel density; iLVD, intra-tumoral lymphatic vessel density.

Table II. P-value comparison of pLVD in PC, NETP, SPTP and $\mathrm{CP}$.

\begin{tabular}{lcccc}
\hline Pancreatic tumor & PC & NETP & SPTP & CP \\
\hline PC & - & 0.000 & 0.011 & 0.027 \\
NETP & 0.000 & - & 0.000 & 0.000 \\
SPTP & 0.011 & 0.000 & - & 1.000 \\
CP & 0.027 & 0.000 & 1.000 & - \\
\hline
\end{tabular}

PC, pancreatic carcinoma; NETP, neuroendocrine tumor of the pancreas; SPTP, solid pseudopapillary tumor of the pancreas; CP, cystadenoma of the pancreas; pLVD, peri-tumoral lymphatic vessel density.
Table III. P-value comparison of iLVD in PC, NETP, SPTP and CP.

\begin{tabular}{lcccc}
\hline Pancreatic tumor & PC & NETP & SPTP & CP \\
\hline PC & - & 0.274 & 0.704 & 0.999 \\
NETP & 0.274 & - & 0.123 & 0.250 \\
SPTP & 0.704 & 0.123 & - & 0.994 \\
CP & 0.999 & 0.250 & 0.994 & - \\
\hline
\end{tabular}

PC, pancreatic carcinoma; NETP, neuroendocrine tumor of the pancreas; SPTP, solid pseudopapillary tumor of the pancreas; CP, cystadenoma of the pancreas; iLVD, intra-tumoral lymphatic vessel density.

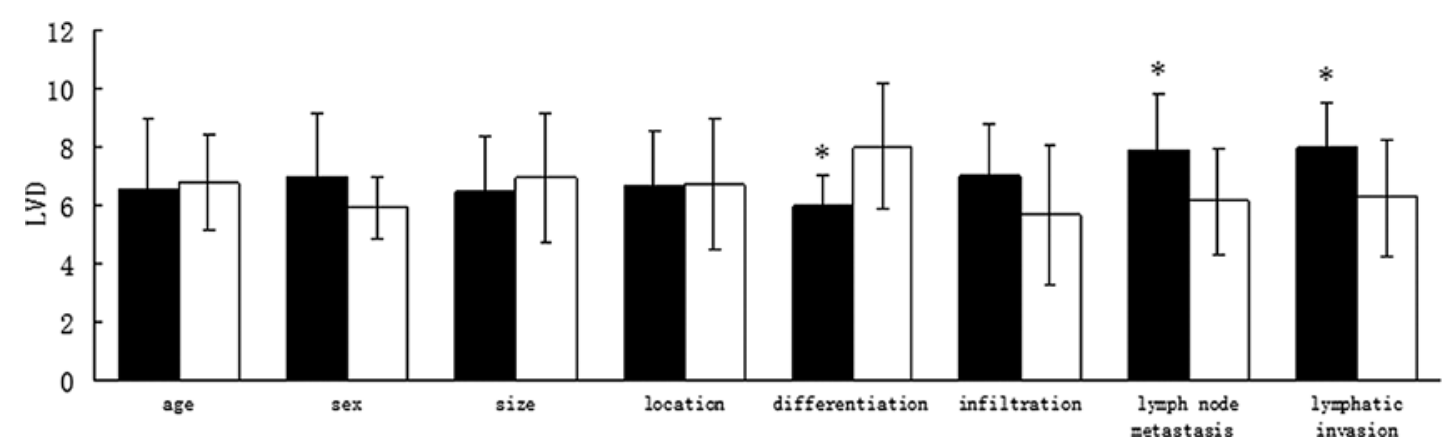

Figure 2. Relationship histogram between peri-tumoral lymphatic vessel density (pLVD) and intra-tumoral lymphatic vessel density (iLVD) of pancreatic cancer (PC) and clinicopathological parameters. Black bars, pLVD of PC; white bars, iLVD of PC. "Statistical difference $(\mathrm{P}<0.05)$.

\section{Discussion}

Lymphatic vessels are the main pathways for the spread of tumor cells to regional lymph nodes $(3,4,8-12)$. The shape of lymphatic vessels is very similar to that of small veins, making it difficult to distinguish lymphatic from blood vessels when they are invaded and destroyed (8). For this reason, lymphatic vessel research lags far behind that of vascular vessels.

Regarding the study of lymphangiogenesis and lymphatic metastasis, the vital problem is the identification of lymphatic vessels. Without a doubt, it is necessary to identify specific cell markers of lymphatic endothelium. A large number of lymphatic vessel markers have been identified in recent years (2,5-7,13), including VEGF-C, VEGF-D, VEGFR-3, LYVE-1, podoplanin, D2-40, Prox-1 and 5'-nucleotidase, which has revealed a new direction for lymphatic vessel research. Among them, the D2-40 monoclonal antibody is generally considered the ideal cell marker of lymphatic endothelium, since it can identify a type of sialoglycoprotein, which is expressed in lymphatic but not vascular endothelium (8).

Detection of lymphangiogenesis by assessing the pLVD and $i L V D$ of $P C$. Previous studies have found that there are no lymphatic vessels in the central area of PC and that pLVD is higher than iLVD in para-tumoral areas of PC (normal tissue areas of the pancreas). According to various researchers, the lymphatic vessels in the peri-tumor tissues of PC are dilated, but PC lymphatic vessels in inter-tumor tissues are occlusive 
and non-functioning $(14,15)$. The immunohistochemical results of our research showed that D2-40 is expressed solely in lymphatic endothelium cells, but not in small vascular vessels. Our study also demonstrated that pLVD is higher than iLVD in PC, and that the shape of pLVD is irregular and dilated. This finding indicates that lymphatic vessel generation of $\mathrm{PC}$ is mainly located in the surrounding tissue of the tumor. Several authors consider the unceasing increase in the amount of tissue and the mechanical pressure due to tumor cell differentiation and proliferation as the main causes of collapse and atrophy in lymphatic vessels in the inter-tumors of PC $(14,16,17)$. Another cause may be the direct invasion and destruction of lymphatic vessel nets by tumor cells $(12,15)$, leading to the decrease in their quantity. With the growth and the development of tumors, the pressure of interstitial tissue gradually increases and causes the opening and the distension of lymphatic capillaries, which were originally in a static state, finally leading to a growth in the number of lymphatic vessels in the peri-tumor area. Meanwhile, the expression of VEGF-C and other regulating factors of lymphatic vessel development increases and leads to abnormal division and proliferation of lymphatic endothelium cells, eventually inducing serious hyperplasia of the peri-tumor tissue of PC.

Comparison of the LVD between PC and other types of pancreatic tumors. We found no difference in iLVD among PC, NETP, SPTP and CP, while there was a great difference in pLVD among PC, NETP, SPTP and CP. The pLVD of NETP was markedly higher than that of the other 3 groups and pLVD of PC was higher than that of SPTP and CP. No difference was found between the two groups of SPTP and CP cases. This result shows that there is no correlation between the iLVD of pancreatic tumors and whether the tumor is benign or malignant. The overwhelming majority of lymphatic vessels in intra-tumor tissues were non-functional. Therefore, the pLVD of a tumor is of marked significance. This conclusion is also consistent with the findings of other authors $(3,8)$.

The pLVD of NETP was significantly higher than that of PC, SPTP and CP, and the reason may be that NETP is a type of tumor with a rich blood supply. Owing to the similarities in the structure and growth pattern of lymphatic and vascular vessels, NETP also induces lymphatic vessel hyperplasia concurrently with angiogenesis. Therefore, the rich pLVD of NETP does not prepare for the lymphatic metastasis of neoplasms, but is solely the result of lymphatic vessel proliferation of NETP following angiogenesis. However, further study of the lymph vessel proliferation mechanism of NETP is required.

$\mathrm{PC}$ is a highly malignant tumor and early metastasis often occurs $(5,14,18,19)$. The lymphatic vessels are the main pathways for tumor spread and metastasis. Therefore, we presumed that the increase in PC pLVD may lead to an increase in the invasion of lymphatic vessels by cancer cells, thus providing the necessary conditions for cancer cell metastasis, via lymphatic vessels.

SPTP and CP are characterized as benign or borderline neoplasms, and metastasis never or seldom occurs. Therefore, lymphatic vessels in peri-tumor tissue may grow in number merely because an increase in mechanical pressure of the interstitial tissue stimulates the proliferation of the resting lymphocapillary vessels. Similar to NETP, SPTP and CP may lack expression of the generating regulatory factors of lymphatic vessels such as VEGF-C. In order to confirm these hypotheses, further study is required.

Relationship between lymphangiogenesis in the peri-tumor tissues of PC and clinicopathological parameters. Various studies have reported the relationship between malignant tumor lymphangiogenesis and clinicopathological parameters. Arigami et al (12) argued that lymphatic vessel invasion is correlated with lymph node micrometastasis in gastric cancer. Other authors $(3,5,20)$ have reported that $\mathrm{pLVD}$ of several types of cancers is correlated with clinical stage, lymphatic vessel invasion and lymph node metastasis. They also suggested that the number of lymphatic vessels in a tumor is not only related to lymphatic vessel metastasis, but is also closely related to a tumor's degree of malignancy, hematogenous metastasis, recurrence and prognosis.

In the present study, the pLVD of PC was correlated with the degree of cancer differentiation, lymphatic node metastasis and lymphatic vessel invasion. The pLVD in well and moderately differentiated PC was lower than that in poorly differentiated and undifferentiated PC. The pLVD in PC with lymphatic node metastasis and lymphatic vessel invasion was significantly higher than that in PC without lymphatic node metastasis and lymphatic vessel invasion. The pLVD in PC was not correlated with age, gender, tumor size, lesion location and peri-pancreas invasion in PC patients. Therefore, we can assert that lymphangiogenesis in the peri-tumor tissue of PC plays a major role in the growth and development of PC. However, iLVD of PC is not correlated with these clinicopathological parameters, which further confirms that lymphatic vessels in the intra-tumor tissues of PC are non-functioning. These findings indicate that detection of the pLVD of PC may predict lymphatic node metastasis and prognosis of PC patients. Meanwhile, this result indicates that restraining lymphangiogenesis in the peri-tumor tissue of $\mathrm{PC}$ is beneficial for obstructing lymphatic node metastasis and may also contribute to the improvement of patient prognosis and the prolongation of patient survival.

\section{Acknowledgements}

This study was supported by the National Science Foundation Program in China (nos. 30870689 and 81071195), and the Jiangsu Province Natural Science Foundation Program in China (no. BK2008325).

\section{References}

1. Harrell MI, Iritani BM and Ruddell A: Tumor-induced sentinel lymph node lymphangiogenesis and increased lymph flow precede melanoma metastasis. Am J Pathol 170: 774-786, 2007.

2. Raica M, Cimpean AM, Ceausu R and Ribatti D: Lymphatic microvessel density, VEGF-C, and VEGFR-3 expression in different molecular types of breast cancer. Anticancer Res 31: 1757-1764, 2011.

3. Kandemir NO, Barut F, Bektas S and Ozdamar SO: Can lymphatic vascular density be used in determining metastatic spreading potential of tumor in invasive ductal carcinomas? Pathol Oncol Res: July, 2011 (E-pub ahead of print).

4. Lin JY, Li XY, Dong P and Nakashima T: Prognostic value of lymphangiogenesis in supraglottic laryngeal carcinoma. J Laryngol Otol 125: 945-951, 2011. 
5. Schulz P, Fischer C, Detjen KM, et al: Angiopoietin-2 drives lymphatic metastasis of pancreatic cancer. FASEB J 25: 3325-3335, 2011.

6. Lee AS, Kim DH, Lee JE, et al: Erythropoietin induces lymph node lymphangiogenesis and lymph node tumor metastasis. Cancer Res 71: 4506-4517, 2011.

7. Klasa-Mazurkiewicz D, Jarzab M, Milczek T, Lipinska B and Emerich J: Clinical significance of VEGFR-2 and VEGFR-3 expression in ovarian cancer patients. Pol J Pathol 62: 31-40, 2011.

8. Kozlowski M, Naumnik W, Niklinski J, Milewski R, Lapuc G and Laudanski J: Lymphatic vessel invasion detected by the endothelial lymphatic marker D2-40 (podoplanin) is predictive of regional lymph node status and an independent prognostic factor in patients with resected esophageal cancer. Folia Histochem Cytobiol 49: 90-97, 2011

9. Kitadai Y, Kodama M, Cho S, et al: Quantitative analysis of lymphangiogenic markers for predicting metastasis of human gastric carcinoma to lymph nodes. Int J Cancer 115: 388-392, 2005.

10. Hall FT, Freeman JL, Asa SL, Jackson DG and Beasley NJ Intratumoral lymphatics and lymph node metastases in papillary thyroid carcinoma. Arch Otolaryngol Head Neck Surg 129: 716-719, 2003.

11. Van den Eynden GG, van der Auwera I, van Laere SJ, et al: Distinguishing blood and lymph vessel invasion in breast cancer: a prospective immunohistochemical study. Br J Cancer 94: 1643-1649, 2006

12. Arigami T, Natsugoe S, Uenosono Y, et al: Lymphatic invasion using D2-40 monoclonal antibody and its relationship to lymph node micrometastasis in pN0 gastric cancer. Br J Cancer 93: 688-693, 2005.
13. Rubbia-Brandt L, Terris B, Giostra E, Dousset B, Morel P and Pepper MS: Lymphatic vessel density and vascular endothelial growth factor-C expression correlate with malignant behavior in human pancreatic endocrine tumors. Clin Cancer Res 10: 6919-6928, 2004.

14. Sipos B, Kojima M, Tiemann K, et al: Lymphatic spread of ductal pancreatic adenocarcinoma is independent of lymphangiogenesis. J Pathol 207: 301-312, 2005.

15. Bailey JM, Mohr AM and Hollingsworth MA: Sonic hedgehog paracrine signaling regulates metastasis and lymphangiogenesis in pancreatic cancer. Oncogene 28: 3513-3525, 2009.

16. Schneider M, Buchler P, Giese N, et al: Role of lymphangiogenesis and lymphangiogenic factors during pancreatic cancer progression and lymphatic spread. Int J Oncol 28: 883-890, 2006.

17. Von Marschall Z, Scholz A, Stacker SA, et al: Vascular endothelial growth factor-D induces lymphangiogenesis and lymphatic metastasis in models of ductal pancreatic cancer. Int J Oncol 27: 669-679, 2005

18. Gaida MM, Haag N, Gunther F, et al: Expression of A disintegrin and metalloprotease 10 in pancreatic carcinoma. Int $\mathrm{J}$ Mol Med 26: 281-288, 2010.

19. Theodoropoulos GE, Panoussopoulos GS, Michalopoulos NV, et al: Analysis of the stromal cell-derived factor 1-3'A gene polymorphism in pancreatic cancer. Mol Med Rep 3: 693-698, 2010.

20. Shields JD, Borsetti M, Rigby H, et al: Lymphatic density and metastatic spread in human malignant melanoma. Br J Cancer 90: 693-700, 2004 